\title{
Multi-task Learning Deep Neural Networks For Speech Feature Denoising
}

\author{
Bin Huang ${ }^{1}$, Dengfeng $\mathrm{Ke}^{2}$, Hao Zheng ${ }^{2}$, Bo X $u^{2}$, Yanyan $\mathrm{Xu}^{1}$, Kaile $\mathrm{Su}{ }^{3}$ \\ ${ }^{1}$ School of Information Science and Technology, Beijing Forestry University, Beijing, China \\ ${ }^{2}$ Institute of Automation, Chinese Academy of Sciences, Beijing, China \\ ${ }^{3}$ Institute for Integrated and Intelligent Systems, Griffith University, Brisbane, Australia \\ fantasyhb@gmail.com, dengfeng.ke@ia.ac.cn, hzheng@nlpr.ia.ac.cn, \\ xubo@ia.ac.cn, xuyanyan@bjfu.edu.cn, k.su@griffith.edu.au
}

\begin{abstract}
Traditional automatic speech recognition (ASR) systems usually get a sharp performance drop when noise presents in speech. To make a robust ASR, we introduce a new model using the multi-task learning deep neural networks (MTL-DNN) to solve the speech denoising task in feature level. In this model, the networks are initialized by pre-training restricted Boltzmann machines (RBM) and fine-tuned by jointly learning multiple interactive tasks using a shared representation. In multi-task learning, we choose a noisy-clean speech pair fitting task as the primary task and separately explore two constraints as the secondary tasks: phone label and phone cluster. In experiments, the denoised speech is reconstructed by the MTL-DNN using the noisy speech as input and it is respectively evaluated by the DNN-hidden Markov model (HMM) based and the Gaussian Mixture Model (GMM)-HMM based ASR systems. Results show that, using the denoised speech, the word error rate (WER) is respectively reduced by $53.14 \%$ and $34.84 \%$ compared with baselines. The MTL-DNN model also outperforms the general single-task learning deep neural networks (STL-DNN) model with a performance improvement of $4.93 \%$ and $3.88 \%$ respectively.

Index Terms: multi-task learning, feature denoising, deep neural networks
\end{abstract}

\section{Introduction}

Speech denoising has become increasingly important for building a robust automatic speech recognition (ASR) system, since the noise presenting in speech makes the accuracy drop down in the ASR system. This has sparked a lot of related researches on speech noisy reduction and speech enhancement.

Traditional mathematical methods are mainly focused on statistical relationship between noise and speech, such as spectral subtraction, Wiener filtering, wavelet speech enhancement, subspace based methods and so on [1, 2, 3, 4]. These methods substantially analyze the shallow statistics of stationary noise in speech under the assumption that noisy and speech are separable, which limits their generalizations.

In recent years, the deep neural network (DNN) has promoted performance on speech recognition accuracy [5], since the DNN has a better ability of capturing the essential and abstract structure of targets. There has also been recent work on speech enhancement and speech denoising using deep autoencoder (DAE) [6,7], which has obtained better performance than the traditional mathematical methods. In these methods, the DAE is trained by greedy layer-wise pre-training and fine- tuning. In the DAE's training of each layer, noisy-clean speech pairs are used as input and output. Therefore, the DAE can be treated as a sort of single-task learning (STL)-DNN and its unique task is minimizing the difference between noisy-clean speech pairs.

Though the STL-DNN performs well in the speech denoising task, they only take advantage of low level speech information without any other assistant constraints. They can be improved by jointly learning multiple related tasks, which is called multi-task learning in machine learning.

Multi-task learning attempts to jointly learn additional interactive tasks to augment the primary task and has been applied to various areas $[8,9,10]$. The DNN architecture can be used for multi-task learning in several different ways which are appealing approaches to well leverage data. It has been previously reported that the multi-task learning (MTL)-DNN can improve performance in speech and language related tasks [11, 12, 13]. In multi-task learning, it is always technical to choose interactive tasks and effectively combine them with existed STL systems.

In this paper we propose a new MTL-DNN model applied to the task of speech denoising for a robust ASR system. In this model, the MTL-DNN is trained with the primary task of noisy-clean speech pair fitting. Meanwhile, two candidate secondary tasks are explored to augment this primary task. One of the secondary tasks is the phone label classification. In this task, the networks are supposed to predict extra phone labels of input noisy-clean speech pairs. Each noisy-clean speech pair is grouped according to its phone label. It enables the networks to learn phonetic structures in noisy-clean speech pairs and substantially increases the phonetic discrimination of reconstructed speech. The other secondary task is the phone cluster classification. It extends the phone label task with relaxed constraints. Each noisy-clean speech pair is divided into several phone clusters instead of one phone label. The phone clusters consisting of phone labels are manually built based on phonology knowledge.

The rest of the paper is organized as follows. In section 2 , we introduce the multi-task learning. Section 3 describes the model structure and training procedures of the proposed MTLDNN model. The experiments and results are given in Section 4. Finally we make conclusions and point out the future work in section 5 .

\section{Multi-task learning}

The multi-task learning approach attempts to solve the primary task by jointly learning multiple related tasks using a shared 
representation. When these additional tasks have been properly organized, it achieves better performance than general single task learning methods.

In this work, in order to augment the primary task of noisyclean speech pair fitting, we explore two secondary tasks: phone label and phone cluster classification.

\subsection{Noisy-clean speech pair fitting task}

In this paper, our goal is to denoise speech features. It can be done by directly mapping noisy speech features to clean ones via neural networks. To this end, we minimize the difference between the networks' output and the expected clean speech. tion:

A Mean Square Error (MSE) is taken as the objective func-

$$
E_{1}=\frac{1}{2} \sum_{i=1}^{N}\left\|\boldsymbol{a}_{i}-\boldsymbol{f}_{i}\right\|^{2}
$$

where $N$ is the number of frames, and $\boldsymbol{f}_{i}$ is the $i$-th frame clean speech feature. $\boldsymbol{a}_{i}$ represents the networks' output vector:

$$
\boldsymbol{a}_{i}=\boldsymbol{W} \cdot \boldsymbol{z}_{i}
$$

where $\boldsymbol{z}_{i}$ is the output vector of the DNN' penultimate layer, and $\boldsymbol{W}$ is this layer's weight matrix.

The derivative of $E_{1}$ wrt. $\boldsymbol{a}_{i}$ is as follows:

$$
\frac{\partial E_{1}}{\partial \boldsymbol{a}_{i}}=\boldsymbol{a}_{i}-\boldsymbol{f}_{i}
$$

\subsection{Phone label task}

The phone label classification is a higher level task compared with the feature pair fitting one. Each phone label represents a specified phonetic collection. In this paper, we focus on Mandarin speech denoising. A Mandarin phone list is shown in Table 1 . The phone list contains 36 vowels and 24 consonants.

We apply one-hot encoding to the phone label targets. The $k$-th bit is set when the input speech frame belongs to the $k$-th phone label.

During propagation, the posterior probability of the $j$-th phone label of the $i$-th speech frame gets evaluated via softmax:

$$
y_{i, j}=\frac{\exp \left(a_{i, j}\right)}{\sum_{j=1}^{D} \exp \left(a_{i, j}\right)}
$$

where $D$ is the dimensionality of phone label targets.

We use the Cross-Entropy (CE) as the objective criterion between the networks' output and the phone label targets:

$$
E_{2}=-\sum_{i}^{N} \sum_{j}^{D} t_{i, j} \log y_{i, j}
$$

where $t_{i, j}$ is the $j$-th phone target of the $i$-th speech frame, and the Cross-Entropy can be differentiated wrt. vector $\boldsymbol{a}_{i}$ as:

$$
\frac{\partial E_{2}}{\partial \boldsymbol{a}_{i}}=\boldsymbol{y}_{i}-\boldsymbol{t}_{i}
$$

\subsection{Phone cluster task}

The phone cluster task is an extension of the phone label task. It takes into consideration the phonological knowledge. As is shown in Table 2, each phone cluster contains several phone labels sharing similar phonological characteristics.
Table 1: Phone label list of Mandarin.

\begin{tabular}{|c|cc|}
\hline Vowel & \multicolumn{2}{|c|}{ Consonant } \\
\hline \hline a ai an & $\mathrm{b}$ & $\mathrm{c}$ \\
ang ao e & $\mathrm{ch}$ & $\mathrm{d}$ \\
ei en eng & $\mathrm{f}$ & $\mathrm{g}$ \\
er i ia & $\mathrm{h}$ & $\mathrm{j}$ \\
ianiang iao & $\mathrm{k}$ & $\mathrm{l}$ \\
ib ie if & $\mathrm{m}$ & $\mathrm{n}$ \\
in ing iong & $\mathrm{p}$ & $\mathrm{q}$ \\
iou o ong & $\mathrm{r}$ & $\mathrm{s}$ \\
ou u ua & $\mathrm{sh}$ & $\mathrm{t}$ \\
uai uan uang & $\mathrm{w}$ & $\mathrm{x}$ \\
uei uen uo & $\mathrm{y}$ & $\mathrm{z}$ \\
van ve vn & $\mathrm{v}$ & $\mathrm{zh}$ \\
\hline
\end{tabular}

Notice that since one speech frame may be divided into several phone clusters, using the one-hot encoding for the multiple targets is not feasible. We propose a block encoding method using two bits to represent the phone cluster states. The active phone clusters are encoded by $(1,0)$, otherwise $(0,1)$. The propagation is slightly different from the one in the phone label task, and the posterior probability of the $c$-th phone cluster of $i$-th speech frame is as follows:

$$
y_{i, c, j}=\frac{\exp \left(a_{i, c, j}\right)}{\sum_{k \in 0,1} \exp \left(a_{i, c, k}\right)}, \forall j=0,1
$$

where $a_{c, i, j}$ is the networks' output corresponding to the $i$-th speech frame, and $c$ is the frame's phone cluster. The objective criterion becomes:

$$
E_{3}=-\sum_{i=1}^{N} \sum_{c=1}^{N_{c}} \sum_{j \in 0,1} t_{i, c, j} \log y_{i, c, j}
$$

where $N_{c}$ is the total number of clusters, and $t_{i, c, j}$ is the corresponding target.

The derivative of $E_{3}$ wrt. $\boldsymbol{a}_{i}$ is:

$$
\frac{\partial E_{3}}{\partial \boldsymbol{a}_{i}}=\boldsymbol{y}_{i}-\boldsymbol{t}_{i}
$$

\section{Training an MTL-DNN Denoising Model}

The proposed MTL-DNN denoising model is a multi-layer perception (MLP) with hidden sigmoid neurons. The structure of the model is shown in Figure 1. The networks in this model consist of two parts: the task independent hidden layers and the multi-task dependent output layers. The hidden layers are initialized by pre-training, and then the whole networks are finetuned with multi-task learning.

\subsection{Pre-training DNN}

Randomly initialized multi-layer networks often get stuck in local optimum in gradient-based training. Therefore, we use a common pre-training method called the Restricted Boltzmann Machine (RBM) to initialize the hidden layers. Each pair of layers in the network can be treated as a RBM, which can be trained using an objective criterion of contrastive divergence [14]. In the pre-training stage, we use the noisy speech as the RBMs' input. 
Table 2: Phone clusters of Mandarin. The vowels in front of '-' mean leading vowels, otherwise trailing vowels.

\begin{tabular}{|c|l|}
\hline Cluster & Phonemes \\
\hline \hline $\mathrm{a}_{-}$ & a ai an ang ao \\
\hline a & a ia ua \\
\hline $\mathrm{e}_{-}$ & e ei en eng er \\
\hline e & e ie ue \\
\hline i_$_{-}$ & i ia ian iang iao ib ie if in ing iong iou \\
\hline i & i ai ei uai uei \\
\hline o_$_{-}$ & o ong ou \\
\hline$\_$o & o ao uo iao \\
\hline $\mathrm{u}_{-}$ & u ua uai uan uang uei uen uo \\
\hline u & u iou ou \\
\hline v & v van ve vn \\
\hline Nasal & $\begin{array}{l}\text { n an ang en eng ian iang in ing iong ong } \\
\text { uan uang uen van vn }\end{array}$ \\
\hline Labial & b p m f \\
\hline Apical & d t n l \\
\hline Velar & g k h \\
\hline Palatal & j q x \\
\hline Spirant & zh ch sh r z c s \\
\hline Other & y w \\
\hline
\end{tabular}

\subsection{Fine-tuning with Multi-task learning}

The structure used for fine-tuning is shown in Figure 1. After pre-training, an affine layer is appended to the output layer of RBMs. This layer is shared by the primary and the secondary tasks. What's more, in the secondary tasks, another softmax layer is appended to the shared affine layer and the softmax transformations are different between these secondary tasks.

In the primary task of noisy-clean speech pair fitting, the output of the affine layer is directly used as the reconstructed feature fitting to the expected clean speech feature and they have the same dimensionality.

In the secondary tasks, the networks have to predict the posterior probabilities of phone labels or phone clusters, so a softmax layer is appended to the shared affine layer. However, the softmax transformations are different between the phone label task and the phone cluster task. In the phone label task, the

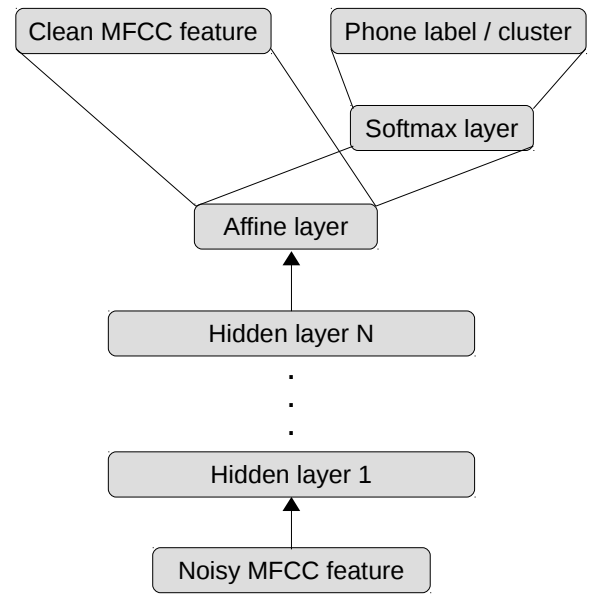

Figure 1: The MTL-DNN denoising model structure. softmax operation is performed on all output units of the affine layer, whereas in the phone cluster task, we perform softmax operations on blocks of the affine layer's output units separately. Each block corresponds to a specified phone cluster. The output part of the secondary task is dropped out in testing.

The networks are fine-tuned by back propagation with minibatch Stochastic Gradient Descent (SGD) algorithm. Notice that different error criteria are applied in the primary task and the secondary task. The primary task is a typical fitting task and uses a Minimum Mean Squared Error (MMSE) criterion, while the secondary classification task uses a Minimum Cross Entropy (MCE) criterion. The dimensions between the MMSE and the MCE are much different. We present a combination method as follows:

$$
E=(1-\alpha) \cdot W_{p} \cdot E_{p}+\alpha \cdot W_{s} \cdot E_{s}
$$

where $E$ is the error sum of the networks. $E_{p}$ and $E_{s}$ indicate the primary task error and the secondary task error respectively. $\alpha$ is a trade-off weight between the primary task and the secondary task. $W_{p}$ and $W_{s}$ are balance weights to solve the dimension mismatch problem.

We can deduce the derivative of $E$ wrt. $\boldsymbol{a}_{i}$ as:

$$
\frac{\partial E}{\partial \boldsymbol{a}_{i}}=(1-\alpha) \cdot W_{p} \cdot \frac{\partial E_{p}}{\partial \boldsymbol{a}_{i}}+\alpha \cdot W_{s} \cdot \frac{\partial E_{s}}{\partial \boldsymbol{a}_{i}}
$$

We choose a form of $W_{p}$ and $W_{s}$ as:

$$
\begin{aligned}
W_{p} & =\sqrt{\frac{1}{N_{m}} \sum_{j \in M}\left\|\frac{\partial E_{s}}{\partial \boldsymbol{a}_{j}}\right\|^{2}} \\
W_{s} & =\sqrt{\frac{1}{N_{m}} \sum_{j \in M}\left\|\frac{\partial E_{p}}{\partial \boldsymbol{a}_{j}}\right\|^{2}}
\end{aligned}
$$

where $M$ is a mini-batch training set with a size of $N_{m}$. Applying (3), (6), (9), to (11), (12) and (13), we get:

$$
\begin{gathered}
W_{p}=\sqrt{\frac{1}{N_{m}} \sum_{j \in M}\left\|\boldsymbol{y}_{j}-\boldsymbol{t}_{j}\right\|^{2}} \\
W_{s}=\sqrt{\frac{1}{N_{m}} \sum_{j \in M}\left\|\boldsymbol{a}_{j}-\boldsymbol{f}_{j}\right\|^{2}} \\
\frac{\partial E}{\partial \boldsymbol{a}_{i}}=(1-\alpha) \cdot W_{p} \cdot\left(\boldsymbol{a}_{i}-\boldsymbol{f}_{i}\right)+\alpha \cdot W_{s} \cdot\left(\boldsymbol{y}_{i}-\boldsymbol{t}_{i}\right)
\end{gathered}
$$

where $W_{p}$ and $W_{s}$ can be automatically updated during the back propagation.

\section{Experiments and results}

\subsection{Data preparation}

Experiments are conducted on a Mandarin corpus. This corpus is contributed by 48 different native students with 11069 utterances. We build two types of corpora: a clean speech corpus and a noisy speech corpus. The clean speech is recorded in quiet background with a $16 \mathrm{kHz}$ sample rate and a mono channel while the noisy speech is made up by adding random road and bus noise to the clean one. The training set consists of 43 speakers and 8600 utterances while the test set is built with 5 speakers and 2469 utterances.

We use the 39 dimensional Mel-frequency cepstral coefficient (MFCC) feature with a $20 \mathrm{~ms}$ frame window and a 10 $\mathrm{ms}$ frame shift. A splice operation that concatenates adjacent 
5 frames with the central frame is performed on the raw input MFCC feature, which results in a 429 dimensional feature vector. [15]

All the experiments are conducted using the Kaldi toolkit

\subsection{Baseline systems}

The baseline systems consist of a Gaussian mixture model and hidden Markov model (GMM-HMM) ASR system and a DNNHMM ASR system.

The GMM-HMM ASR system using 2500 senones is trained on the clean training set. It is used to generate forced alignments for training DNN and obtaining frame-level phone label information. In the DNN-HMM ASR system, we use a shared HMM trained in the GMM-HMM ASR system. The DNN is configured with 5 hidden layers and 1024 hidden units in each layer and is pre-trained using the RBM method. We use a learning rate of 0.008 in the fine-tuning stage. The DNNHMM ASR system is also trained on clean test set.

Using the word error rate (WER) as the criterion, we evaluate the performance of the trained DNN-HMM and GMMHMM ASR systems on noisy test set as baselines.

\subsection{MTL-DNN denoising model}

The necessary phone label information of each frame is obtained via the previously trained HMM triphone system. Several MTL-DNN models are trained on the noisy training set. They use the same schedule with the DNN-HMM system in pre-training the DNN. We choose 0.00001 as the start learning rate and use the learning rate halving strategy.

In order to evaluate the performance of the MTL-DNN model, we reconstruct the denoised test set by passing the noisy test set to the trained MTL-DNN denoising system and then test it on the previously trained DNN-HMM and GMM-HMM ASR systems respectively.

\subsection{Effect of the secondary task weight}

We first explore the relationship between the WER and the secondary task weight. From (10) we learn that the secondary task weight $\alpha$ determines how much the secondary task affects the networks. When $\alpha$ is larger than 0.5 , the secondary task is dominant and becomes the primary task. Figure 2 shows the curve of WER of DNN-HMM ASR along with the secondary task weight ranging from 0.1 to 0.9 . In the phone label MTL-DNN, the WER of DNN-HMM baseline system reaches the minimum of $21.03 \%$ at 0.2 , while in the phone cluster MTL-DNN the minimum of $21.28 \%$ at 0.5 . There is an increasing trend of WER with the secondary weight growing, which verifies our hypothesis above.

\subsection{Performance comparison}

The experimental results of performance comparison are shown in Table 3. The baseline DNN-HMM and GMM-HMM systems have a WER of $9.36 \%$ and 16.95 on the clean MFCC test set respectively. However, both of their performance sharply drop on the noisy MFCC test set and get a WER of $44.88 \%$ and $47.19 \%$ respectively.

The DNN based denoising models including STL-DNN and MTL-DNN significantly improve the performance. Using the denoised speech, the WER of the DNN-HMM baseline is relatively reduced by $50.71 \%-53.14 \%$, while the WER of the GMM-HMM baseline is relatively reduced by $31.72 \%-34.84 \%$.

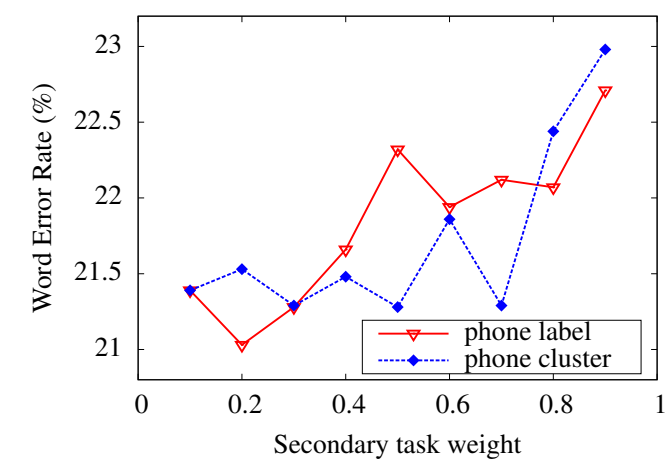

Figure 2: Relationship between the Word Error Rate (WER) and the secondary task weight in MTL-DNNs using different secondary tasks.

Besides, the MTL-DNN model using the phone label as the secondary task outperforms the STL-DNN model using noisyclean speech pair fitting as the unique task with a relative improvement of $4.93 \%$ and $3.88 \%$ on the WER of the DNN-HMM system and the GMM-HMM system respectively. It shows the superior effectiveness of our proposed MTL-DNN model in the speech denoising task.

Table 3: The WER (\%) of the DNN-HMM system and the GMMHMM system with different types of features. The secondary task weights selected for the phone label and the phone cluster task are 0.2 and 0.5 respectively.

\begin{tabular}{|c|c|c|c|}
\hline Feature & Task & GMM-HMM & DNN-HMM \\
\hline \hline Clean MFCC & - & 16.95 & 9.36 \\
\hline Noisy MFCC & - & 47.19 & 44.88 \\
\hline STL-DNN & Pair fitting & 31.99 & 22.12 \\
\hline MTL-DNN & Phone label & $\mathbf{3 0 . 7 5}$ & $\mathbf{2 1 . 0 3}$ \\
\hline MTL-DNN & Phone cluster & 32.22 & 21.28 \\
\hline
\end{tabular}

\section{Conclusions}

In this paper, we propose a new MTL-DNN method and apply it to the speech denoising task. In the proposed model, we design a primary task of noisy-clean speech pair fitting and explore two secondary tasks: phone label and phone cluster classification. Experiments are conducted to analyze the relationship between the primary task and the secondary task. Experimental results of performance comparison prove the superior effectiveness of our proposed MTL-DNN model. It significantly reduces the WER of baseline systems and outperforms the STL-DNN model in speech denoising task.

In the future, we will try to integrate the denoising module into the ASR system and investigate other related secondary tasks such as state-level posteriors and Gauss-level posteriors.

\section{Acknowledgements}

This work is supported by the National 973 program in China (No.2013CB329302), the National Natural Science Foundation of China (61472369), the Fundamental Research Funds for the Central Universities (YX2014-18), and the Beijing Higher Education Young Elite Teacher Project (YETP0768). 


\section{References}

[1] S. Boll, "Suppression of acoustic noise in speech using spectral subtraction," IEEE Transactions on Acoustics, Speech and Signal Processing, pp. 113-120, 1979.

[2] J Meyer, and K. U. Simmer, "Multi-channel speech enhancement in a car environment using Wiener filtering and spectral subtraction", IEEE International Conference on Acoustics, Speech and Signal Processing, pp. 1167-1170, 1997

[3] M. Bahoura, and J Rouat, "Wavelet speech enhancement based on the Teager energy operator," Signal Processing Letters, IEEE, pp. 10-12, 2001.

[4] F Asano, S Hayamizu, T. Yamada, et al., "Speech enhancement based on the subspace method," IEEE Transactions on Speech and Audio Processing, pp. 497-507, 2000.

[5] G. Hinton, L. Deng, D. Yu, et al., "Deep neural networks for acoustic modeling in speech recognition: The shared views of four research groups," Signal Processing Magazine, IEEE, pp. 82-97, 2012.

[6] X. Feng., Y. Zhang, and J. Glass, "Speech feature denoising and dereverberation via deep autoencoders for noisy reverberant speech recognition," IEEE International Conference on Acoustics, Speech and Signal Processing, pp. 1759-1763, 2014.

[7] X. Lu, Y. Tsao, S. Matsuda, et al., "Speech enhancement based on deep denoising autoencoder," INTERSPEECH, pp. 436-440, 2013.

[8] G. Obozinski, B. Taskar, and M. Jordan, "Multi-task feature selection,” Statistics Department, UC Berkeley, Tech. Rep, 2006.

[9] O. Chapelle, P. Shivaswamy, S. Vadrevu, et al., "Multi-task learning for boosting with application to web search ranking," Proceedings of the 16th ACM SIGKDD international conference on knowledge discovery and data mining, pp. 1189-1198, 2010.

[10] X. Wang, C. Zhang, and Z. Zhang, "Boosted multi-task learning for face verification with applications to web image and video search," IEEE conference on computer vision and pattern recognition, pp. 142-149, 2009.

[11] M. L. Seltzer, J. Droppo, "Multi-task learning in deep neural networks for improved phoneme recognition," IEEE International Conference on Acoustics, Speech and Signal Processing, pp. 69656969, 2013.

[12] D. Chen, B. Mak, C. C. Leung, et al., "Joint acoustic modeling of triphones and trigraphemes by multi-task learning deep neural networks for low-resource speech recognition," IEEE International Conference on Acoustics, Speech and Signal Processing, pp. 55925596, 2014.

[13] R. Collobert, and J. Weston, "A unified architecture for natura language processing: Deep neural networks with multitask learning," Proceedings of the 25th international conference on Machine learning, pp. 160-167, 2008.

[14] G. Hinton, "A practical guide to training restricted boltzmann machines," Neural Networks: Tricks of the Trade Lecture Notes in Computer Science, vol. 7700, pp. 599-619, 2012.

[15] Povey, Daniel and Ghoshal, Arnab and Boulianne, et al., "The Kaldi Speech Recognition Toolkit," IEEE Signal Processing Society, 2011. 